\title{
TRANSIENT BIT ERROR RATE ANALYSIS OF DATA RECOVERY SYSTEMS USING JITTER MODELS*
}

\author{
Yonghui Tang and Randall L. Geiger \\ Department of Electrical and Computer Engineering \\ Iowa State University, Ames, IA 50010, U.S.A
}

\begin{abstract}
This paper presents a method for analyzing the Bit Error Rate of recovered data for PLL-based data recovery systems (DRS) as the PLL comes into lock. This method is based on the analyses of the transient response of the Phase-Locked Loop (PLL) and the associated jitter models. It provides a means to predict the acquisition time needed for a data recovery system to reach a given BER. Practical criteria are established to help determine the "lock" status of a PLL.
\end{abstract}

\section{INTRODUCTION}

Demand is increasing for even higher speed serial data links. A key component in these data links is a data recovery system. The overall BER performance of these links is dominantly determined by the characteristics of the data recovery system. High-speed low-power phaselocked loops are an integral part of data/clock recovery system. Although the performance of the PLL after it is in lock is reasonably well understood, its performance during lock acquisition has received minimal attention in the literature but is also of concern since this determines how long it will take for a PLL to attain an acceptable BER.

The performance of the data recovery system is usually characterized by the Bit Error Rate (BER) of the recovered data. The BER is determined by the jitter of the incoming data and the jitter performance of the PLL. In this paper, we develop the relationship between the BER of the recovered data and the jitter of the incoming data both when the PLL is in lock and when the PLL is acquiring lock.

A typical PLL-based data recovery system is shown in figure 1. Research on PLL has been ongoing for decades and the term "lock" is widely used to indicate the PLL is in a special "steady state" mode of operation. But until now, a rigorous definition of "lock" has not been presented in the literature. It is generally assumed that a PLL is in lock when the output of the loop filter stabilizes and that one just "knows" when the PLL is in lock but, in reality, the control voltage for the VCO comes from a loop filter that generally has an infinite impulse response and, as such, only asymptotically approaches a steady-state value or a steady-state average value. In this work, a practical criteria for determining if the PLL is in "lock" will be developed and this "lock" condition will be contingent upon establishing a given BER level of performance.

To determine if a data recovery system is working correctly, we usually establish a maximum acceptable value for the BER, denoted in this work as $B_{a c c}$. If we assume that the frequency of the incoming data does not change for time $t>t_{0}$, then if the BER of the recovered data satisfies the relationship $B E R<B_{a c c}$ for time all $t>t_{1}$, where $t_{1}>t_{o}$, then we say the PLL is in "lock" for $t>t_{1}$. If $t_{1}$ is the minimum value of $t$ for which the BER satisfies the inequality $B E R\left(t_{1}\right) \leq B_{a c c}$, then we say the PLL acquires lock at time $t_{1}$.

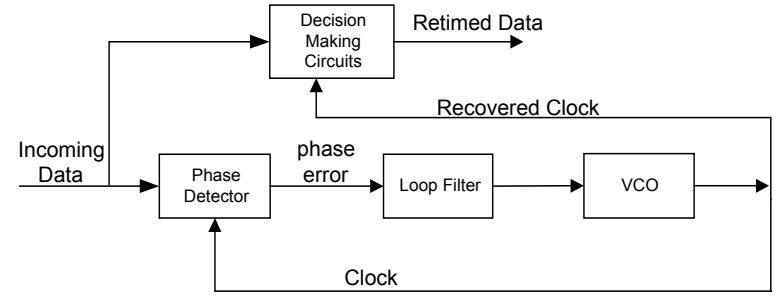

Figure 1. PLL-based data recovery system

In the following sections, we will analysis the BER of the recovered data based on the acquisition behavior of the PLL and the jitter model.

\section{ACQUISITION BEHAVIOR OF THE PLL}

The acquisition behavior of the PLL can be studied most conveniently by considering the response of the loop to an

\footnotetext{
${ }^{*}$ This work was supported, in part, by NSF, SRC and National Semiconductor Inc.
} 
initial phase error or a frequency error. Consider the common second-order PLL shown in figure 2.

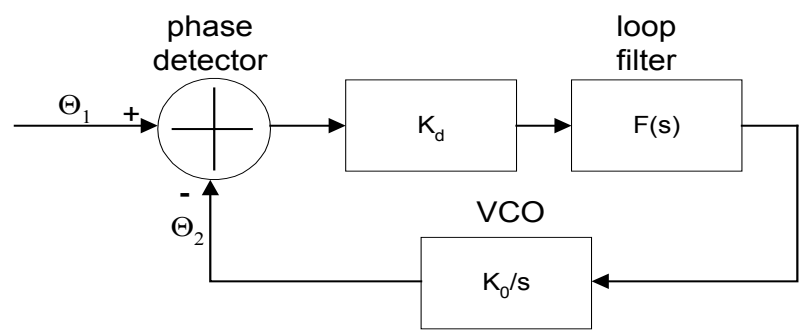

Figure 2. Common second-order PLL

Assume that the phase detector is linear. The transfer function of the second-order PLL is given by

$$
H(s)=\frac{\Theta_{2}(s)}{\Theta_{1}(s)}=\frac{K_{0} K_{d} F(s)}{s+K_{0} K_{d} F(s)}
$$

The phase error transfer function is given by

$$
H(s)=\frac{\Theta_{e}(s)}{\Theta_{1}(s)}=\frac{s}{s+K_{0} K_{d} F(s)}
$$

where $K_{d}$ is the phase detector gain, $K_{o}$ is the VCO gain and the $F(s)$ is the transfer function of the loop filter.

The acquisition process of the PLL is classified into two distinct types, lock-in process and pull-in process.

Assuming initially the PLL is in lock, the lock-in process is the re-acquisition process during which the output of the phase detector will only sweep once within its output range before the PLL returns to lock. Pull-in is the re-acquisition process during which the output of the phase detector will sweep within its output range more than one time before the PLL returns to lock. The pull-in process is more complicated and takes much longer time than the lock-in process and it is a highly nonlinear process. The typical control voltage response of the lock-in process and the pull-in process are illustrated in figure 3 .
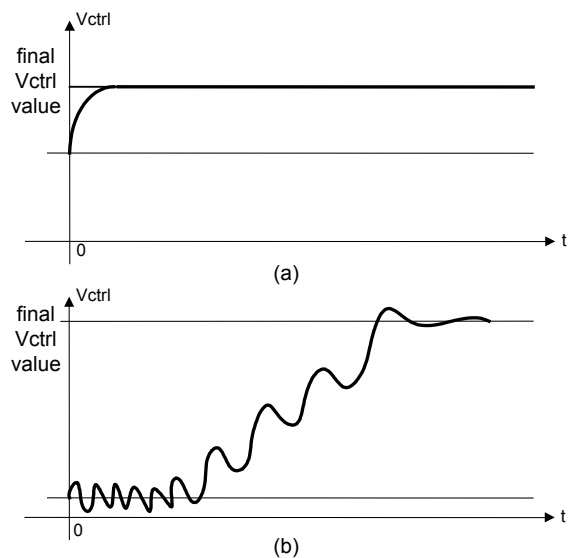

Figure 3. (a) Lock-in process (b) Pull-in process

\section{JITTER AND ITS MODEL}

Jitter is the deviation from the ideal timing of an event. It is composed of both deterministic and random (Gaussian) components.

The deterministic jitter is the jitter with a nonGaussian probability density function. It is always bounded in amplitude and has specific causes. Deterministic jitter is characterized by its bounded, peak-to-peak value.

Random jitter is the jitter that is characterized by a Gaussian distribution. It is defined to be the peak-to-peak value which is given to be 14 times the standard deviation of the Gaussian distribution for a BER of $10^{-12}$.

In the following, we will define the jitter models which will generate plots of eye closure vs. BER with various amount of random and deterministic jitter components.

The error probability is defined as

$$
P(Q)=\frac{1}{2}\left(1-\operatorname{erf}\left(\frac{Q}{\sqrt{2}}\right)\right)
$$

where $\operatorname{erf}(\mathrm{)})$ is the error function which is given by

$$
\operatorname{erf}(x)=\frac{2}{\sqrt{\pi}} \int_{0}^{x} e^{-t^{2}} d t
$$

and $\mathrm{Q}$, the average signal to noise ratio, is defined as

$$
Q=\frac{1}{2}\left(\frac{V}{\sigma}\right)
$$

where $\mathrm{V}$ is the peak to peak signal amplitude and $\sigma$ is the root-mean-square noise. To arrive at this expression, it is assumed that the noise has a Gaussian probability density function with zero mean.

\section{A. Effects Of Random Jitter}

Let $Q T_{1}$ be the ratio of the eye opening to the amount of random jitter at an eye crossing, i.e.

$$
Q T_{1}\left(T_{0}, t, \sigma\right)=\frac{1}{2}\left(\frac{T_{0}}{\sigma}\right)
$$

To include the effect of sampling time, $Q T_{1}$ can be rewritten as $Q T_{1}\left(T_{0}, t, \sigma\right)=\frac{1}{2}\left(\frac{T_{0}+|t|}{\sigma}\right)$

where $t$ is a dummy variable that defines the offset of the sampling instant from the eye crossing. When $\mathrm{t}=0$, a worstcase $\mathrm{BER}$ is obtained, i.e. $\mathrm{t}=0$ defines the position of the eye crossing.

If the decision threshold is made at the eye crossing, then the eye opening is essentially, zero, i.e. $T_{0}=0$.

Following the analysis in the signal domain, the BER in the time domain is defined as

$$
P T_{1}\left(T_{0}, t, \sigma\right)=\frac{1}{2}\left(1-\operatorname{erf}\left(\frac{Q T_{1}\left(T_{0}, t, \sigma\right)}{\sqrt{2}}\right)\right)
$$


In order to study the eye closure, let us define the position of the second eye crossing. The second eye crossing would have similar characteristics as the first one and occurs a bit period away, i.e.,

$Q T_{2}\left(T_{0}, t, \sigma\right)=Q T_{1}\left(T_{0}, t-|T|, \sigma\right)$

Similarly, $P T_{2}\left(T_{0}, t, \sigma\right)=\frac{1}{2}\left(1-\operatorname{erf}\left(\frac{Q T_{2}\left(T_{0}, t, \sigma\right)}{\sqrt{2}}\right)\right)$

The BER now is given by

$$
P\left(T_{0}, t, \sigma\right)=P T_{1}\left(T_{0}, t, \sigma\right)+P T_{2}\left(T_{0}, t, \sigma\right)
$$

\section{B. Effects of Deterministic Jitter}

Deterministic jitter (DJ) is caused by varying patterns or duty cycle creating predominant spectral components or DC baseline drift in the transmitted signal. DJ reduces the eye width and can be assumed to have larger amplitude than random jitter. To account for DJ, both $\mathrm{QT}_{1}$ and $\mathrm{QT}_{2}$ can be written as

$$
Q T_{1}\left(T_{0}, t, \sigma, D J\right)=\frac{\left(T_{0}-D J\right)+|t|}{2 \sigma}
$$

Similarly, $Q T_{2}\left(T_{0}, t, \sigma, D J\right)=Q T_{1}\left(T_{0}, t-|T|, \sigma, D J\right)$

The BER is now

$$
P T_{1}\left(T_{0}, t, \sigma, D J\right)=\frac{1}{2}\left(1-\operatorname{erf}\left(\frac{Q T_{1}\left(T_{0}, t, \sigma, D J\right)}{\sqrt{2}}\right)\right)
$$

and

$$
P T_{2}\left(T_{0}, t, \sigma, D J\right)=\frac{1}{2}\left(1-\operatorname{erf}\left(\frac{Q T_{2}\left(T_{0}, t, \sigma, D J\right)}{\sqrt{2}}\right)\right)
$$

The total probability over the window of interest is therefore

$$
P\left(T_{0}, t, \sigma, D J\right)=P T_{1}\left(T_{0}, t, \sigma, D J\right)+P T_{2}\left(T_{0}, t, \sigma, D J\right)
$$

\section{Total Jitter Model}

A complete jitter model due to the total jitter can be obtained by combining the random jitter model and the deterministic jitter model together.

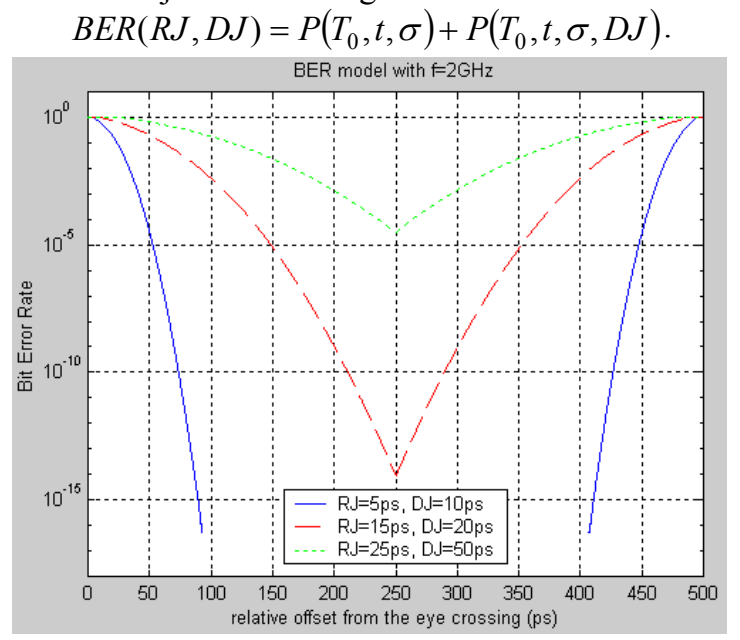

Figure 4. BER with different RJ and DJ combination
The effects of different combination of the random jitter and deterministic jitter are shown in figure 4.

\section{BER ANALYSIS OF THE DATA RECOVERY SYSTEM}

Once we have obtained the jitter models and the acquisition behavior of the PLL. We can use them to calculate the transient BER during acquisition or even at anytime.

As before, we can get an optimal BER when the clock samples the data at the middle between the two eye crossings. This is also the principle for the decisionmaking circuits. In the decision-making circuits, the incoming data should be re-sampled by the recovered clock with sampling edges at the middle of a bit period.

We assume that initially the PLL is in lock with reference signal with frequency $\omega_{0}$. At $\mathrm{t}=0$, there is a frequency step $\Delta \omega$ applied to the reference signal. After the step, the angular frequency of the reference becomes $\omega_{1}(t)=\omega_{0}+\Delta \omega u(t)$; the phase of the reference signal $\phi_{1}(t)$ is the integral over the frequency variation $\Delta \omega$. So that $\phi_{1}(t)=\Delta \omega t$.

From the transient response of the VCO, specifically from the output $\omega_{2}(t)$, we can get the phase of the VCO output $\phi_{2}(t)$ which is given by $\phi_{2}(t)=\int_{0}^{t}\left|\omega_{2}(\tau)-\omega_{0}\right| d \tau$.

The phase difference is then given by $\Delta \phi=\left|\phi_{1}-\phi_{2}\right|$. In order to study the position of the sampling edges within the eye pattern, we need to know the relative phase shift within the two eye crossings which is given by

$$
\Delta \phi_{2 \pi}=\Delta \phi \bmod 2 \pi, \Delta \phi_{2 \pi} \in(0,2 \pi) .
$$

The situation when $\Delta \phi_{2 \pi}=\pi$ means that the sampling edges are in the middle of the eye pattern, this is the best case for BER.

The situation when $\Delta \phi_{2 \pi}=0$ or $2 \pi$ means that the sampling edges are at the eye crossings, this is the worst case for BER.

By knowing the phase difference between the reference signal (signal to be re-sampled) and the regenerated clock, combined with the jitter models, the BER can be precisely calculated.

\section{THEORETICAL ANALYSIS EXAMPLE}

To demonstrate this method, a PLL shown in figure 5 was analyzed for calculating the BER during acquisition.

Some specifications of the PLL and the data recovery system are as follows:

1. Phase detector is a sinusoid phase detector,

$$
U_{d}=K_{0} \sin (\Delta \phi), K_{0}=3
$$

2. Loop filter is a passive loop filter 
3. Initial PLL locking frequency $=2 \mathrm{GHz}$, frequency step $=20 \mathrm{MHz}$

4. VCO gain $K_{0}=3.5 \times 10^{8}$ radians $/ V$

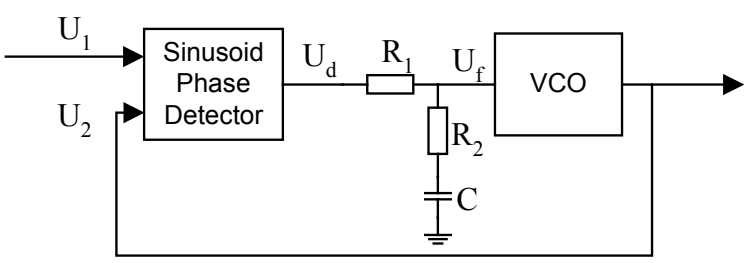

Figure 5. Structure of PLL for simulation

Using above parameters for the PLL and combining with the jitter model with deterministic jitter $=20 \mathrm{pS}$ and random jitter $=15 \mathrm{pS}$, the following simulation results were obtained.

Figure 6(a) shows the transient response of the phase detector and loop filter outputs. The output of the phase detector swept the output range many times until it merged with the output of the loop filter. It's a pull-in process because of the large frequency step.

Figure 6(b) shows the corresponding BER during the acquisition. At the early stages of the acquisition, the BER changes dramatically with a large range. During this time, the BER is unacceptably large. When the PLL approaches lock, the BER drops steeply. Figure 6(c) shows the magnified BER response. From this figure, we see that after about $\mathrm{t}=6.242 \mu \mathrm{S}$, the BER dropped below $1 \times 10^{-12}$.

This example does not include jitter of the PLL because the transient analysis of the acquisition process is ideal. However, it can provide an easy and quick method to approximately evaluate a data recovery system.

This example shows an application of this method on early (behavioral level design) design stage. It is also applicable for after-design verification. When the data recovery system design is completed, combining the transient simulation results and the jitter models, we can get results that are very close to the real world.

\section{CONCLUSION}

For a data recovery system, the BER can be calculated by using the jitter model and the transient response of the PLL. This makes it possible to predict when the data recovery system will enter lock.
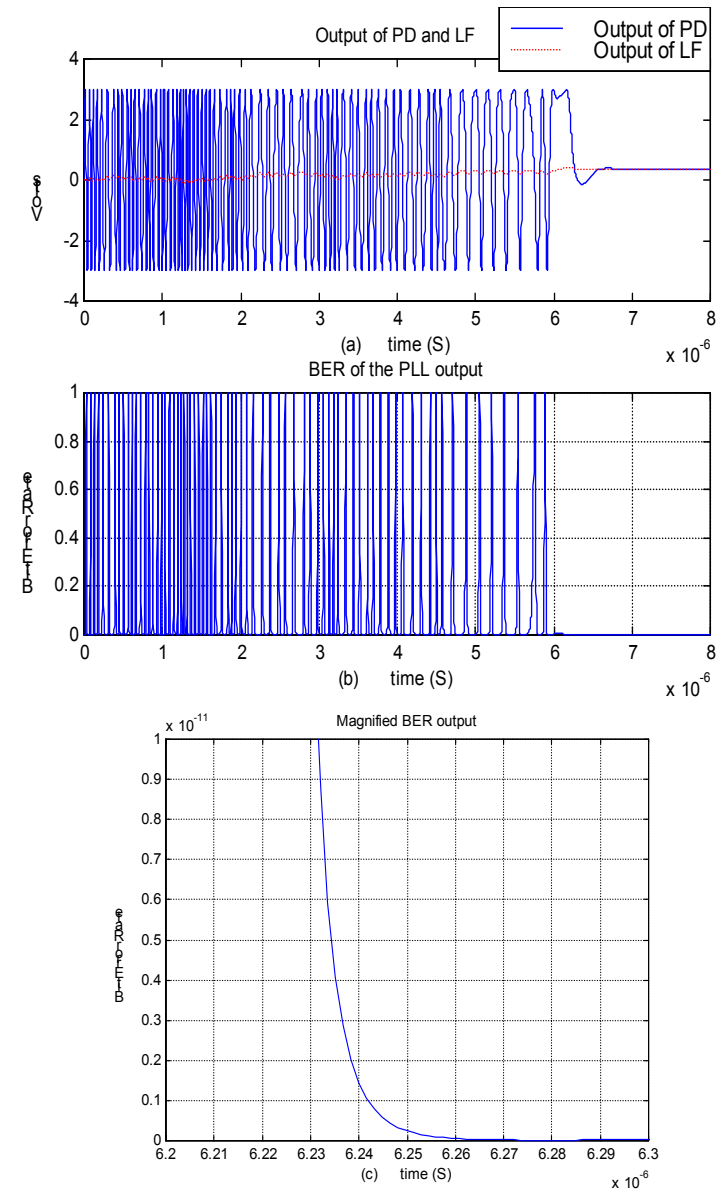

Fig. 6 Simulation results (a) Transient response of the PLL (b) Transient value of BER (c) Magnified BER response

\section{REFERENCES}

[1] "Phase-locked loops: design, simulation, and applications", $3^{\text {rd }}$ edition, Roland E. Best, McGraw Hill, 1997

[2] "Phase-locked loop circuit design", Dan H. Wolaver, Prentice Hall, 1991.

[3] "Phaselock techniques", $2^{\text {nd }}$ edition, Floyd Martin Gardner, John Wiley \& Sons, Inc. 1979

[4] "Information Technology-Fibre Channel - Methodologies for Jitter Specification”, Working Draft, T11.2 / Project 1230/ Rev 2.0, February 8, 1998 